\title{
EL TRABAJO A DISTANCIA EN ESPAÑA: ANÁLISIS EN CLAVE PREVENTIVA TRAS LA APROBACIÓN DE LA LEY 10/2021, DE 9 DE JULIO ${ }^{1}$
}

Remote work in Spain: analysis from a preventive perspective following the approval of Law $10 / 2021$, of July 9

\author{
FRANCISCA FERNÁNDEZ PROL ${ }^{2}$ \\ Universidade de Vigo
}

\begin{abstract}
RESUMEN
Resumen: En el marco de la crisis sanitaria, configurado el trabajo a distancia como opción prioritaria, este ha alcanzado una dimensión sin precedentes en España, en no pocos casos en circunstancias de extrema precariedad. Con útiles domésticos, en despachos improvisados y al tiempo que se atendían obligaciones de crianza y cuidado, trabajadores al uso - presenciales- se han convertido, de la noche a la mañana, en trabajadores a distancia o, de modo más preciso, en teletrabajadores. Tras meses de pandemia y recién aprobada la norma que procura responder a un escenario tan excepcional, un diagnóstico de situación resulta necesario. La Ley 10/2021, de 9 de julio, es, a continuación, objeto de análisis en clave preventiva, confrontando sus aportaciones en la materia a los riesgos específicos y peculiaridades del trabajo a distancia.
\end{abstract}

\section{PALABRAs CLAVE}

Trabajo a distancia, teletrabajo, prevención de riesgos laborales.

\section{ABSTRACT}

Abstract: In the context of the health crisis, with telework as a priority option, remote work has reached an unprecedented level, in many cases in extremely precarious circumstances. With household supplies, in makeshift offices and while providing for upbringing and care, regular workers -face-to-face workers- have become, overnight, remote workers or, more precisely, teleworkers. After months of pandemic and recently approved the rule that seeks to respond to such an exceptional scenario a diagnosis of the situation is necessary. Law 10/2021, of July 9, is then object of preventive analysis, comparing its contributions in the matter to the specific risks and peculiarities of remote work.

\section{KEYWORDS}

Remote work, telework, prevention of occupational risks.

\section{Introducción: un modelo de luces y sombras}

Hace ya un tiempo que apreciamos un resurgimiento del trabajo a distancia, ahora, en la mayoría de los casos, en su versión tecnológica, bajo la modalidad de teletrabajo. A ello han contribuido la irrupción y universalización de las tecnologías de la información y la comunicación (en adelante, TICs), pero también sus ventajas en términos organizativos, para empresarios y asimismo trabajadores, e incluso en clave colectiva. Empezando por esto último, el trabajo a distancia sin duda conlleva beneficios desde una perspectiva medioambiental -habida cuenta la reducción de los desplazamientos y consecuentemente de las emisiones vinculadas a estos- y en

\footnotetext{
1 Trabajo realizado en el marco del Proyecto DER2017-83488-C4-3-R "Los derechos fundamentales ante el cambio del trabajo subordinado en la era digital", financiado por el Ministerio de Ciencia, Innovación y Universidades.

2 Profesora Titular de Derecho del Trabajo y de la Seguridad Social. Universidade de Vigo, Vigo, España. Correo electrónico:
} franfernandez@uvigo.es. 
términos de empleo -al propiciar el desarrollo de actividades desde zonas alejadas de los núcleos urbanos en que acostumbra concentrarse la demanda de mano de obra, así como al facilitar el acceso al mercado de trabajo de colectivos con especiales dificultades, por ejemplo por su movilidad reducida-. Ya desde la perspectiva empresarial, muchos ensalzan el ahorro de costes o la flexibilidad -en particular, horaria- propiciados por este modo de prestación de servicios. Flexibilidad, por otro lado, a menudo muy apreciada por los trabajadores, que encuentran en la misma una valiosa herramienta para la conciliación de la vida personal, familiar y laboral -aún, como se verá, no exenta de sombras, señaladamente desde la perspectiva del principio de igualdad entre mujeres y hombres-.

Y la crisis sanitaria generada por la Covid-19 ha puesto de manifiesto una nueva virtud del trabajo a distancia: su capacidad para mantener, en ciertos casos en términos prácticamente equivalentes, la actividad de no pocas empresas y sectores, aún cerrados estos últimos y confinados los trabajadores. En tiempos de pandemia, el trabajo a distancia se erige en escudo protector de la salud de los trabajadores, que, exentos de desplazarse a su lugar de trabajo, no se exponen a riesgos de contagio. Por otro lado, al mantener la actividad, el trabajo a distancia permite, así mismo, preservar los empleos, virtualidad sin duda de primer orden en aquel contexto de parálisis de sectores clave. No en vano, el art. 5 del Real Decreto-ley $8 / 2020^{3}$ insta a la implementación de "sistemas de organización que permitan mantener la actividad (...), particularmente por medio del trabajo a distancia", configurando estos como "prioritarios frente a la cesación temporal o reducción de la actividad" ${ }^{4}$. Una solución de urgencia que, al no revertirse la pandemia, perdura desde hace ya varios meses y se mantiene incluso, ya matizada, tras la reciente Ley de trabajo a distancia ${ }^{5}$.

$Y$, sin embargo, no todo son luces. El trabajo a distancia presenta también importantes sombras. En términos, en primer lugar, de condiciones de trabajo: invisible el trabajador, la ocultación fraudulenta de la relación jurídico-laboral bajo otras fórmulas generadoras de una protección más endeble -mediante el recurso a falsos autónomos- se revela más fácil. Por otro lado, fuera de las instalaciones de la empresa, el control del cumplimiento de la normativa laboral, de modo paradigmático en materia de tiempo de trabajo, se torna más complejo. $Y$ en muchas ocasiones el entorno de trabajo o las herramientas a disposición del trabajador presentan notables insuficiencias, hallándose el trabajador a distancia en condiciones sensiblemente más precarias que el trabajador presencial, con el consiguiente impacto en términos de formación o promoción profesional del primero. Ya en el caso del trabajo a distancia por razones de conciliación, la predisposición de las trabajadoras -frente a los trabajadores- a la adaptación de sus condiciones laborales por razones de cuidado, permite anticipar un impacto de género y, consiguientemente, una previsible discriminación por razón de sexo -por la mayor exposición femenina a las disfunciones típicas de todo trabajo a distancia y, además, a aquellas en cambio propias del trabajo a distancia por razones de conciliación-. Finalmente, la asunción por el trabajador de gastos inherentes al desarrollo de su actividad-de manera señalada, en el marco del trabajo a distancia sobrevenido como consecuencia de la crisis sanitaria- ha determinado una ruptura del equilibrio económico del contrato.

Capítulo aparte merece el impacto del trabajo a distancia en la salud del trabajador objeto del presente estudio-. Las condiciones materiales de desempeño de la actividad, la flexibilidad característica de la misma o la hiperconexión, en el caso del teletrabajo, generan riesgos específicos, cuya prevención, en el domicilio o lugar señalado por el trabajador, se revela por otro lado especialmente dificultosa. Más aún habida cuenta, la relajación operada por la

\footnotetext{
${ }^{3}$ Real Decreto-ley 8/2020, de 17 de marzo, de medidas urgentes extraordinarias para hacer frente al impacto económico y social del COVID-19.

${ }^{4}$ Interpretando el referido art. 5: CRUZ (2020b), p. 408; CASAS (2020), p. 921; ÁLVAREZ (2020), p. 192.

${ }^{5}$ Prueba de ello es la Disp. Trans. 3a de la Ley 10/2021, de 9 de julio, de trabajo a distancia, a cuyo tenor: "Al trabajo a distancia implantado excepcionalmente en aplicación del artículo 5 del Real Decreto-ley 8/2020, de 17 de marzo, o como consecuencia de las medidas de contención sanitaria derivadas de la COVID-19, y mientras estas se mantengan, le seguirá resultando de aplicación la normativa laboral ordinaria". Ello, con todo, sin perjuicio de la obligación empresarial de dotación de medios, equipos, herramientas y consumibles y de su mantenimiento.
} 
norma de urgencia ya referida -art. 5 del Real Decreto-ley 8/2020-, al entender cumplida, "la obligación de efectuar la evaluación de riesgos, en los términos previstos en el art. 16 de la Ley 31/1995, de 8 de noviembre, de Prevención de Riesgos Laborales [en adelante LPRL], con carácter excepcional, a través de una autoevaluación realizada voluntariamente por la propia persona trabajadora" ${ }^{\prime}$. La regla, en aquel excepcional escenario de emergencia, era comprensible y legítima, al fundamentarse en el objetivo de prevención de un riesgo inminente de probable mayor impacto: el contagio por Covid-19. Con todo, transcurridos unos meses y con la vista puesta en el medio y largo plazo, la intervención del legislador resultaba, por razones preventivas y de otra índole -alguna más arriba apuntada-, perentoria ${ }^{7}$.

La respuesta, en este caso, adoptó la forma de una "norma sustantiva única" y "transversal", fruto, además, de un previo diálogo social materializado en el Acuerdo de Trabajo a Distancia: el Real Decreto-ley 28/2020, de 22 de septiembre, posteriormente convertido en Ley $10 / 2021$, de 9 de julio, de trabajo a distancia ${ }^{8}$. En dicha norma se halla hoy la definición de este último -así como de la subcategoría de teletrabajo-, su régimen jurídico -a efectos, en particular, de acceso y de tránsito entre las modalidades presencial y no presencial-, así como la relación de derechos que asisten al trabajador a distancia. De todos ellos, es a continuación objeto de estudio su derecho a la prevención de riesgos laborales. A tal efecto, la nueva regulación será confrontada a los riesgos específicos del trabajo a distancia en general y del teletrabajo en particular, prestando especial atención a aquellos derivados de la ordenación del factor tiempo de trabajo. Así mismo, el análisis abordará las dificultades inherentes a la proyección de la actividad preventiva en el domicilio del trabajador o en el lugar libremente elegido por este para la prestación de servicios.

\section{El nuevo marco normativo: la Ley $10 / 2021$, de 9 de julio, de trabajo a distancia}

El trabajo a distancia es objeto de un Convenio -y de una Recomendación- de la OIT, de un Acuerdo Marco Europeo y ahora, a nivel interno, de una Ley, prestando tales normas una atención desigual a la prevención de riesgos laborales.

El primero, el Convenio 177 (1996), sobre el trabajo a domicilio ${ }^{9}$-no ratificado por España-, aboga por el fomento de la igualdad de trato respecto de los trabajadores presenciales "en particular, respecto de la protección en materia de seguridad y salud en el trabajo" -art. 4-, establece la aplicación de las legislaciones internas en la materia al "trabajo a domicilio", "teniendo en cuenta las características propias de este", y conmina a los Estados "a determinar las condiciones en que, por razones de seguridad y salud, ciertos tipos de trabajo y la utilización de determinadas sustancias podrán prohibirse en el trabajo a domicilio" -art. 7-. Previsiones objeto de desarrollo por la Recomendación 184 (1996), cuyo apartado VII formula, ya a título orientativo, un breve elenco de obligaciones empresariales y de los trabajadores en materia de seguridad y salud, y establece pautas de actuación ante la concurrencia de un riesgo grave e inminente.

En el marco europeo, la norma de referencia es el Acuerdo Marco Europeo sobre Teletrabajo, de 16 de julio de $2002^{10}$, a cuyo tenor "el empresario es responsable de la salud y la seguridad profesional del teletrabajador, con arreglo a la Directiva 89/391/CEE y a las directivas específicas, las legislaciones nacionales y los convenios colectivos pertinentes". De manera específica y con objeto de "comprobar la correcta aplicación de las disposiciones aplicables en materia de salud y seguridad", el Acuerdo refiere el derecho de acceso del

\footnotetext{
${ }^{6}$ Sobre dicha autoevaluación, CRUZ (2020b), pp. 409-410.

7 Destacando que el teletrabajo "requerirá una mayor densidad reguladora, estatal y colectiva" (...) con un mayor control del tiempo de trabajo o medidas específicas de protección de riesgos laborales", CASAS Y RODRÍGUEZ-PIÑERO (2020), p. 348.

${ }^{8}$ Las expresiones entrecomilladas se extraen de la Exposición de Motivos (apartado IV).

${ }^{9}$ Sobre este, puede consultarse USHAKOVA (2019), pp. 1364-1379, así como COMISIÓN DE EXPERTOS EN APLICACIÓN DE CONVENIOS Y RECOMENDACIONES (2020), p. 209.

${ }^{10}$ Alcanzado por la Confederación Europea de Sindicatos (CES), la Unión de Confederaciones de la Industria y de Empresarios de Europa (UNICE) / la Unión Europea del Artesanado y de la Pequeña y Mediana Empresa (UNICE/UEAPME) y el Centro Europeo de la Empresa Pública (CEEP).
} 
empresario, de los representantes de los trabajadores y/o de las autoridades competentes al lugar del teletrabajo; de tratarse del domicilio del trabajador, previa notificación y acuerdo de este. Finalmente, el Acuerdo expresa el derecho del teletrabajador a "solicitar una visita de inspección".

En España, hasta la reciente reforma, el art. 13 del Real Decreto 2/2015, de 23 de octubre, por el que se aprueba el texto refundido de la Ley del Estatuto de los Trabajadores (en adelante, ET) conformaba el precepto de referencia. Hoy por hoy, tal precepto estatutario se limita a recordar la posibilidad de prestación de servicios a distancia "en los términos previstos en la Ley 10/2021, de trabajo a distancia". Norma que, entre otras muchas novedades, ofrece un concepto nuevo de trabajo a distancia. Así, a tenor de la misma, es tal aquella "forma de organización del trabajo o de realización de la actividad laboral conforme a la cual esta se presta en el domicilio de la persona trabajadora o en el lugar elegido por esta, durante toda su jornada o parte de ella, con carácter regular" (art. 2.a). Circunstancia ${ }^{11}$, a su vez concurrente al prestarse el trabajo a distancia, "en un periodo de tres meses, un mínimo del treinta por ciento de la jornada, o el porcentaje proporcional equivalente en función de la duración del contrato de trabajo" (art. 1). De modo que, en principio, sólo el trabajo a distancia no ocasional, que alcance o supere el umbral referido -abarcando, en su caso, la jornada completa-, queda subsumido en el ámbito de aplicación de la nueva norma. Todo ello, sin embargo, sin perjuicio de lo dispuesto en la materia por la negociación colectiva, dotada, por la norma de 2021 (Disp. Adic. 1áa), de un amplio margen de maniobra, que alcanza incluso a la identificación del trabajo a distancia: así, "Ios convenios o acuerdos colectivos podrán regular (...) un porcentaje o periodo de referencia inferiores a los fijados en la presente ley a los efectos de calificar como 'regular' esta modalidad de ejecución de la actividad laboral". La negociación colectiva, por tanto, puede configurar como trabajo a distancia, con los efectos señalados por la Ley, experiencias más residuales a las inicialmente contempladas por el citado art. 1 de la Ley 10/2021. Incluso, convenios o acuerdos, pueden identificar puestos y funciones susceptibles de desarrollo a distancia -excluyendo, por tanto, otras actividades de dicha modalidad- o limitar temporalmente tal modo de prestación de servicios (de nuevo, Disp. Adic. 1a). Por otro lado, la nueva norma sí mantiene la configuración del trabajo a distancia como macro-categoría comprensiva de subcategorías o especies varias: el trabajo a distancia que cabría calificar como "tradicional" y el teletrabajo, ahora definido expresamente por el legislador como "aquel trabajo a distancia que se lleva a cabo mediante el uso exclusivo o prevalente de medios y sistemas informáticos, telemáticos y de telecomunicación". Por lo que, siguiendo de nuevo el Acuerdo Marco Europeo, el teletrabajo precisa del recurso por el trabajador, de modo exclusivo o preponderante, a dos peculiares herramientas: los equipos informáticos y las TICs ${ }^{12}$.

En lo que atañe a la materia preventiva, la norma de 2021 completa la parca mención del anterior art. 13 ET. Así, por un lado, reitera -por cierto, con una dicción literal prácticamente idéntica-, el derecho de los trabajadores a distancia a "una adecuada protección en materia de seguridad y salud", de nuevo de conformidad con lo previsto por la Ley 31/1995 y de su normativa de desarrollo (art. 15). Reitera, pues, lo obvio, pero no por ello menos relevante: el derecho del trabajador a distancia, ex arts. 4.2.d) y $19 \mathrm{ET}$, a la protección de su integridad física ${ }^{13}$ y su inclusión en el ámbito de aplicación de la normativa preventiva ${ }^{14}$. De modo que, si bien no es este lugar para una exhaustiva revisión del haz de derechos y obligaciones generados al amparo de la normativa preventiva, sí debe dejarse claro que, en cualquier caso, con independencia pues de su explícita plasmación normativa, también el trabajador a distancia

${ }^{11}$ Por cierto, también presente en la definición de teletrabajo del Acuerdo Europeo (art. 2): “el teletrabajo es una forma de organización y/o de realización del trabajo, con el uso de las tecnologías de la información, en el marco de un contrato o de una relación de trabajo, en la que un trabajo que hubiera podido ser realizado igualmente en los locales del empleador, se efectúa fuera de estos locales de manera regular".

12 Para un análisis conceptual más detallado, puede consultarse THIBAULT (2001), pp. 23 y ss.; SIERRA (2011), pp. 29 y ss.; MELLA Y VILLALVA (2015), pp. 47 y ss.; DE LAS HERAS (2016), pp. 29 y ss.; MELLA (2017), pp. 27 y ss.; SALA (2020), pp. 17 y ss.

${ }^{13}$ Derecho, conviene recordarlo, dotado de dimensión constitucional, por su vinculación con el derecho a la vida y a la integridad física (art. 15 de la Constitución Española, en adelante CE), así como con el derecho a la salud (art. $43 \mathrm{CE}$ ).

${ }^{14}$ Conforme a lo indicado por el art. 3 de la Ley 31/1995, en coherencia, a su vez, con la Directiva 1989/391/CEE (arts. 2 y 3 ) 
tiene derecho a una protección eficaz en materia de seguridad y salud (art. 14 LPRL). Pervive, por tanto, al igual que en la modalidad presencial, el correlativo deber empresarial de protección, cuya implementación práctica ha de atender aquellos objetivos (art. 5 LPRL) y principios básicos enumerados por la norma de referencia (art. 15 LPRL): así, entre estos últimos y con la mirada puesta en los trabajadores a distancia, evitar los riesgos, evaluar aquellos que no se pueden evitar, combatir los riesgos en su origen, adaptar el trabajo a la persona, sustituir lo peligroso por lo que entrañe poco o ningún peligro, planificar la prevención o dar las debidas instrucciones a los trabajadores. Así mismo, vigente el deber general de prevención y protección -en cuya virtud, como se recordará, el empresario no sólo ha de cumplir aquellas obligaciones explícitamente previstas por la normativa preventiva, sino también cuantas otras sean necesarias (arts. 14.2 y 3 LPRL)-, perviven igualmente otros deberes empresariales: de información y formación de los trabajadores (arts. 18 y 19 LPRL), de vigilancia de su salud (art. 22 LPRL), o de tutela especial de ciertos grupos de trabajadores, por ejemplo, sujetos, fuera de las instalaciones de la empresa, a un "cierto ritmo" (arts. 25 a 28 LPRL y 36 ET). Y también en el marco del trabajo a distancia, el empresario ha de cumplir aquellas concretas medidas de prevención señaladas por la Ley: así, en síntesis, de evaluación de los riesgos (art. 16 LPRL), de constitución de servicios de prevención (arts. 30 y 31 LPRL), de acondicionamiento de locales y herramientas -temática abordada por Directivas y reglamentos-, de provisión de equipos de protección individual (art. 17 LPRL), así como ante situaciones de emergencia o riesgo grave e inminente (arts. 20 y 21 LPRL). Finalmente, respecto de todo ello, no decaen, en la modalidad no presencial, los derechos de información, consulta y participación de los trabajadores, en su caso a través de los correspondientes órganos de representación (art. 18 LPRL).

Pero la norma de 2021 va más allá que la de 2012. Además del referido art. 15, la Sección 4a del Capítulo III de la Ley 10/2021 acoge otro precepto -art. 16- que atiende a dos aspectos especialmente relevantes. Alude, por un lado, a los riesgos inherentes al desarrollo del trabajo a distancia -riesgos denominados "característicos de esta modalidad de trabajo"- y atiende, por otro, a las peculiaridades de su proceso de evaluación habida cuenta la prestación de servicios fuera de las instalaciones de la empresa, a menudo en el domicilio del trabajador. Además, al margen de la citada Sección -expresamente dedicada al "Derecho a la prevención de riesgos laborales"- otros preceptos son así mismo susceptibles de lectura en clave preventiva. Tal es el caso, por supuesto, de aquellos dedicados a la ordenación del factor tiempo de trabajo (arts. 13 y 14, relativos a los derechos al horario flexible y al registro horario adecuado), o por los que se reconocen los derechos a la desconexión digital (art.18) y a la conciliación y corresponsabilidad (art. 4.5) -habida cuenta, de nuevo, su impacto en la jornada y horario del trabajador-. Pero también, la configuración misma del trabajo a distancia como modalidad de trabajo de "carácter regular" -susceptible de desempeño a jornada completa- o las "limitaciones" señaladas para determinados colectivos - menores a los que se exige una presencia mínima en la empresa (art. 3)- son cuestiones relevantes desde una perspectiva preventiva.

El esfuerzo del legislador, sin duda, es loable, pues los desafíos no son menores. Como es obvio, la aplicación de las normas preventivas "generales" al trabajo desempeñado en el domicilio del trabajador o en el lugar libremente designado por este presenta dificultades, en orden a su implementación y también a su verificación. En cuanto a lo primero, pesa, sin duda, el paradigma del trabajador presencial, cuya protección procura prioritariamente la vigente normativa de seguridad y salud, por ello en esencia enfocada a la evitación y prevención del riesgo en las instalaciones -físicas- titularidad de la empresa o sobre las que el empresario proyecta su poder de dirección ${ }^{15}$. Y precisamente por esto último, el control de la aplicación rigurosa de la normativa preventiva, también se torna más dificultoso en espacios privados ajenos a la empresa. Incluso la mayor autonomía organizativa del trabajador a distancia -a menudo determinando jornada, horarios y descansos, lugares y condiciones de trabajo o herramientas- redunda en decisiones con notable trascendencia en clave preventiva.

${ }^{15}$ CRUZ (2020a), p. 1. 
Además, pueden desarrollarse a distancia prestaciones de servicios sumamente dispares: actividades tradicionales y manuales mediante el recurso a herramientas muy rudimentarias; profesiones altamente cualificadas o empleos tecnológicos, en cambio, precisados de equipos muy sofisticados; o tareas, de complejidad variable, pero efectuadas mediante recursos electrónicos. Y caben, por otro lado, múltiples alternativas organizativas: el volumen de jornada o trabajo desempeñados a distancia puede variar sensiblemente y los servicios pueden prestarse en línea o, por el contrario, sin conexión y, en cualquier caso, con grados de autonomía muy dispares. En particular, en el caso del teletrabajo, son necesarios matices específicos, que atiendan al recurso intensivo a medios informáticos y TICs.

Por si fuera poco, como ya se ha referido, la declaración del estado de alarma y la configuración del trabajo a distancia como prioritario, "por su capacidad potencial de reducir la probabilidad de exposición y contagio por COVID-19", han fundamentado la introducción, vía norma de urgencia, de una excepción de notable calado. "Con carácter temporal", en aquellos sectores, empresas o puestos de trabajo que no contemplasen el trabajo a distancia, la obligación de evaluación de riesgos, ex art. 16 LPRL, resultó sustituida por una autoevaluación realizada voluntariamente por la propia persona trabajadora (art. 5 Real Decreto-Ley 8/2020) ${ }^{16}$. Quienes en los últimos meses se convirtieron, de la noche a la mañana, en trabajadores a distancia, se hallaron, pues, desde una perspectiva preventiva, en una situación de especial vulnerabilidad: pues, no se operó, en términos estrictos, la adaptación de las medidas de prevención "a las modificaciones que puedan experimentar las circunstancias que incidan en la realización del trabajo" (art. 14.2 LPRL). La referida autoevaluación, en efecto, no puede asimilarse a ello, y sólo puede validarse temporalmente, en el contexto de la emergencia sanitaria.

\section{Ponderación crítica a la luz de los riesgos específicos del trabajo a distancia y del teletrabajo}

El trabajo a distancia genera riesgos específicos. A ello atiende, tras la reciente reforma, la normativa española, que apela expresamente a que la evaluación de los riesgos y la planificación de la actividad preventiva tengan en cuenta "los riesgos característicos de esta modalidad de trabajo, poniendo especial atención en los factores psicosociales, ergonómicos y organizativos". Y especial cuidado han de merecer, "en particular", "la distribución de la jornada, los tiempos de disponibilidad y la garantía de los descansos y las desconexiones durante la jornada" (art. 16.1 Ley 10/2021).

La precisión, sin duda, merece acogerse favorablemente, pues contribuye a visibilizar una problemática a menudo ignorada: el domicilio o aquel lugar libremente designado por el trabajador no siempre conforman entornos seguros y condiciones de trabajo y herramientas pueden así mismo generar riesgos singulares, físicos y también psicosociales.

\subsection{Riesgos físicos}

Las condiciones medioambientales y ergonómicas en que se prestan los servicios, así como las herramientas de trabajo empleadas por los trabajadores a distancia son fuentes potenciales de riesgos físicos.

En cuanto a lo primero, ya se trate de teletrabajo o no, las dimensiones del espacio físico en que se desarrollan las funciones, su separación o no del resto de la vivienda, las condiciones de luz, ventilación o ruido, al igual que las posturas adoptadas para el desempeño de la actividad -forzadas o por periodos prolongados- son susceptibles de generar daños en la salud física del trabajador. Por ello, debe plantearse la aplicabilidad de la normativa preventiva en la materia:

\footnotetext{
${ }^{16}$ Determinando "una flexibilización verdaderamente excepcional del plan de prevención existente en la empresa" - CASAS (2020), p 929 - que "no resulta suficiente, ni satisfactoria" - CRUZ (2020b), p. 416-. 
así, en España, del Real Decreto 486/1997, de 14 de abril, por el que se establecen las disposiciones mínimas de seguridad y salud en los lugares de trabajo, por el que se transpone la Directiva $89 / 654 / \mathrm{CEE}^{17}$. Con todo, resulta difícil pasar por alto que las citadas normas, interna y europea, definen, respectivamente, los "lugares de trabajo" como las "áreas del centro de trabajo" o, de manera más restrictiva, como "los lugares destinados a albergar puestos de trabajo, situados en los edificios de la empresa y/ o del establecimiento, incluido cualquier otro lugar dentro del área de la empresa y/ o del establecimiento al que el trabajador tenga acceso en el marco de su trabajo". Circunstancia que debiera impulsar una aclaración por parte del legislador, extendiendo explícitamente el campo de aplicación de la norma referida al trabajo desempeñado a distancia -evidentemente, con las necesarias adaptaciones- o, mejor aún, alumbrando una normativa nueva, dirigida específicamente a la definición de las condiciones medioambientales en que, aún en sus domicilios $u$ otros lugares determinados por los trabajadores a distancia, estos debieran de desempeñar su labor. Más aún, habida cuenta la regulación de las condiciones que ha de reunir, desde una perspectiva preventiva, el entorno de trabajo de los teletrabajadores.

Para estos últimos, en efecto, ha de estarse, aun hoy, al Real Decreto 488/1997, de 14 de abril, sobre disposiciones mínimas de seguridad y salud relativas al trabajo con equipos que incluyen pantallas de visualización, de aplicación generalizada a cualquier trabajador, por consiguiente, presencial o a distancia, "que habitualmente y durante una parte relevante de su trabajo normal utilice un equipo con pantalla de visualización" (art. 2.c). Pues, su anexo identifica aquellas condiciones objetivas en que ha de prestarse, en las instalaciones de la empresa o no, el trabajo con equipos informáticos. Así, por ejemplo, al requerir que el espacio físico en que se desarrolla la actividad tenga una dimensión suficiente, esté dotado de unos niveles adecuados de iluminación y la humedad o ruido se hallen en parámetros razonables (apartado 2, "Entorno" del Anexo).

Y también las herramientas de trabajo son potenciales focos de riesgos físicos, a cuyos efectos debe, por lo de ahora, abogarse, aún con dificultades interpretativas, por la aplicación, también a los trabajadores a distancia, del Real Decreto 1215/1997, de 18 de julio, relativo a la utilización de los equipos de trabajo, así como del Real Decreto 773/1997, de 30 de mayo, en materia de equipos de protección individual. Además, en el caso particular del teletrabajo, el empleo, por periodos muy prolongados -en no pocas ocasiones, a lo largo de toda la jornada-, de equipos informáticos con pantallas de visualización se asocia a la generación de daños oftalmológicos, trastornos musculo-esqueléticos, patologías vinculadas al sedentarismo o carga o fatiga mental. Circunstancia de nuevo objeto de tratamiento específico por el ya citado Decreto 488/1997, que, si bien urge modernizar, no resulta todo él desechable. Merece rescatarse, en primer término, la identificación misma -y consiguiente visibilización- de los riesgos vinculados al manejo de ordenadores, que cabría clasificar en tres categorías: sensoriales, físicos y psicosociales. Y guardan sentido, por supuesto, las obligaciones empresariales consignadas: señaladamente, de evaluación -a cuyos efectos la norma conmina a valorar los tiempos de utilización del equipo y de atención continuada de la pantalla, así como el grado de atención exigido-, pero también, en línea con la normativa general (LPRL), de vigilancia de la salud, así como de información y formación. Ya en el Anexo el Decreto de 1997 enumera los requisitos mínimos exigidos al "equipo de trabajo", comprensivo de pantalla, teclado, mesa o superficie de trabajo y asiento. Así, para prevenir riesgos físicos -en particular, oculares o musculares- indica, por ejemplo, que "la imagen de la pantalla deberá ser estable, sin fenómenos de destellos" o que el asiento deberá "proporcionar al usuario "libertad de movimiento", así como una "postura confortable", siendo su altura regulable o el respaldo reclinable.

El análisis realizado, sin duda, pone de manifiesto la insuficiencia y obsolescencia de la normativa vigente en España. Es necesario, en primer término, regular las condiciones mínimas de seguridad y salud exigibles al lugar de trabajo cuando este no se halle en las instalaciones de

${ }^{17}$ Destacando la aplicación a los trabajadores a distancia del Real Decreto 486/1997, SIERRA (2011), p. 257. 
la empresa, sino en el domicilio del trabajador u otro lugar elegido por este. El entorno de trabajo del trabajador a distancia debe, dicho de otro modo, ser objeto de atención específica. Ya en el caso particular del teletrabajo, resulta perentoria, una reforma modernizadora del Real Decreto 488/1997, que atienda a los avances tecnológicos operados en las últimas décadas, con toda probabilidad generadores potenciales de nuevos riesgos, también físicos, para la salud de los trabajadores: la irrupción de dispositivos informáticos más allá de las simples computadoras -en particular, dispositivos móviles- y, por supuesto, de las TICs, a cuyo amparo surgen nuevos cauces de proyección del poder de dirección y nuevas formas de organización del trabajo.

Y la intervención normativa es particularmente urgente en el presente contexto: con toda probabilidad, numerosos puestos de trabajo improvisados en domicilios tras declararse el estado de alarma no cumplen siquiera con los requisitos básicos hoy por hoy vigentes. Y el retorno a la presencialidad no sólo no parece en muchos casos inmediata, esta no será ya, para numerosos trabajadores, absoluta o a jornada completa. En dicho sentido, la excepción introducida por la Disp. Trans. 3 a de la nueva Ley de trabajo a distancia resulta criticable. Según esta, en efecto, "al trabajo a distancia implantado excepcionalmente en aplicación del art. 5 del Real Decreto 28/2020, de 17 de marzo, o como consecuencia de las medidas de contención sanitaria derivadas de la COVID-19, y mientras estas se mantengan, le seguirá resultando de aplicación la normativa laboral ordinaria". Tras meses de pandemia y con un horizonte a medio plazo poco halagüeño, debiera, también en estos casos y tras un periodo de adaptación razonable, exigirse el ajuste, cuando menos en extremos de especial trascendencia. Así, se ha hecho -párrafo segundo de la citada Disp.- respecto de la obligación empresarial de dotación de medios, equipos, herramientas y consumibles y así debiera haberse hecho en materia preventiva, tiempo de trabajo inclusive.

\subsection{Riesgos psicosociales}

Dos advertencias deben preceder el análisis a continuación acometido. Por un lado, ha de destacarse que si bien el mismo procura una identificación individualizada de los elementos determinantes de los riesgos psicosociales -para contribuir a su visibilización y a la correlativa articulación de medidas preventivas-, lo cierto es que, muy a menudo, estos últimos y las consiguientes patologías o lesiones, surgen de la confluencia o superposición de varias circunstancias: por ejemplo, entornos materialmente poco propicios a la concentración, confusión de tiempos de trabajo y de descanso, aislamiento, sobrecarga de trabajo y requerimiento permanente de la empresa a través de TICs frente a las que el sujeto desarrolla paulatinamente sentimientos de rechazo.

$Y$ debe subrayarse, así mismo, que resulta sumamente complejo predeterminar el impacto en la salud de los trabajadores de los riesgos psicosociales. Este, en efecto, dependerá de las circunstancias profesionales concurrentes -confluencia de múltiples riesgos, tiempos de exposición a los mismos...- pero también, como se verá, de factores personales. Con todo, es frecuente la generación de enfermedades o lesiones psicosomáticas tales como trastornos del sueño y gastrointestinales, así como dolores de cabeza y musculares o el desarrollo del denominado "síndrome del burn out" o "del trabajador quemado".

\subsubsection{Riesgos psicosociales vinculados al tiempo de trabajo}

Desde una perspectiva preventiva, el tiempo de trabajo debe cuidarse especialmente: no en vano las normas en la materia -señaladamente, la Directiva 2003/88/CE- persiguen, en última instancia, "promover la mejora de la seguridad y de la salud de los trabajadores" 18 . Y tal marco normativo, europeo e interno -arts. 40.2 CE y 34 y 38 ET-, es de aplicación, sin excepciones, a los trabajadores a distancia: rigen pues, para estos, el límite, infranqueable, de la jornada

${ }^{18}$ STJUE de 10 de septiembre de 2015, Asunto C-266/14, Tyco; y STJCE de 1 de diciembre de 2005, Asunto C-14/04, Abdelkader Dellas. 
máxima anual - 40 horas semanales de trabajo efectivo de promedio en cómputo anual-, así como aquel, en su caso pactado en convenio colectivo o, en defecto de este, por acuerdo de empresa, de aplicación a la jornada diaria -como es sabido, a falta de los citados acuerdos, de 9 horas diarias ${ }^{19}$. Y los trabajadores a distancia son así mismo acreedores de iguales descansos diarios y semanales- y vacaciones que los trabajadores presenciales. Con todo, no son pocos los problemas aplicativos generados por el distanciamiento físico de las dependencias de la empresa y, más aún, por la flexibilidad inherente a la prestación de servicios a distancia.

El trabajo a distancia plantea, en primer término, un problema de cómputo mismo de la jornada, esto es, de limitación del tiempo de trabajo y, consecuentemente, de salvaguarda de los tiempos de descanso. A tal efecto, en Derecho español, la regla se halla en el art. 34.5 ET, a cuyo tenor, "el tiempo de trabajo se computará de modo que tanto al comienzo como al final de la jornada diaria el trabajador se encuentre en su puesto de trabajo". Y es tiempo de descanso, a su vez, conforme dispone el art. 2.2 de la Directiva 2003/88/CE, aquél que no es de trabajo. Pues, si bien tal norma es aplicable al trabajo a distancia, hallándose entonces el referido puesto de trabajo en el domicilio del trabajador o en el lugar libremente elegido por este, el distanciamiento físico añade dificultades al, de por sí, complejo control del tiempo de prestación de servicios. Una temática, por cierto, en España, objeto de intervención relativamente reciente por Real Decreto-ley $8 / 2019$, de 8 de marzo, por el que, tras un intenso debate judicial, se impone a la empresa la obligación de garantizar "el registro diario de jornada, que deberá incluir el horario concreto de inicio y finalización de la jornada de trabajo de cada persona trabajadora" (art. 34.9 ET). Obligación que, al sustentarse, en palabras del TJUE, en el "derecho fundamental de todo trabajador a la limitación de la duración máxima del tiempo de trabajo y a periodos de descanso diario y semanal" ${ }^{20}$, resulta, a priori, de aplicación universal a todos los trabajadores, cualquiera que sea su sector de actividad y modo de prestación de servicios, inclusive, por tanto, a distancia. Lo que, ahora, es objeto de reconocimiento expreso ex art. 14 de la Ley 10/2021, a cuyo tenor "el sistema de registro horario que se regula en el art. $34.9 \mathrm{ET}$, de conformidad con lo dispuesto en la negociación colectiva, deberá reflejar fielmente el tiempo que la persona trabajadora que realiza trabajo a distancia dedica a la actividad laboral, sin perjuicio de la flexibilidad horaria, y deberá incluir, entre otros, el momento de inicio y finalización de la jornada".

Tal aclaración se estima conveniente. Pues, queda así patente que, también en el caso de los trabajadores a distancia, el control del tiempo de trabajo es responsabilidad del empleador. Sobre el mismo recae, por tanto, la obligación de implementación del correspondiente sistema de registro, de conformidad, en su caso, con los métodos de organización y documentación dispuestos por la negociación colectiva o, en defecto de regulación convencional, previa consulta de estos extremos con los representantes de los trabajadores ${ }^{21}$. Caben, pues, al igual que para los trabajadores presenciales, registros manuales -mediante la introducción de datos en hojas o libros de servicios o programas informáticos por el propio trabajador-, o automáticos -particularmente en el caso de los teletrabajadores, mediante la detección del encendido y apagado del dispositivo electrónico o de la conexión a la correspondiente plataforma o aplicación ${ }^{22}$-. Con todo, al igual que en el caso de los trabajadores presenciales, también en el caso de los trabajadores a distancia -tal vez incluso con mayor facilidad-, la fiabilidad de tales fórmulas de registro puede verse comprometida: bien por la articulación por el empleador de mecanismos de manipulación de los registros o por verse compelido el propio trabajador a confeccionar datos falsos ${ }^{23}$, bien por no registrarse ciertas tareas -por ejemplo, en el caso del teletrabajo, aquellas que no se ejecuten en red o previa conexión del trabajador-.

Pero debe repararse en otra circunstancia añadida: la prestación de servicios fuera de las instalaciones de la empresa, señaladamente en el domicilio del trabajador -en que también

${ }^{19}$ FERNÁNDEZ (2020), p. 262.

${ }^{20}$ STJUE de 14 de mayo de 2019.

${ }^{21}$ FERNÁNDEZ (2020), p. 268.

${ }^{22}$ FERNÁNDEZ (2020), p. 269.

${ }^{23}$ IGUARTUA (2019), p. 147; FERNÁNDEZ (2020), p. 269. 
desarrolla su vida personal y familiar-, difumina las fronteras entre tiempo de trabajo y tiempo de descanso. Siendo el domicilio del trabajador lugar de trabajo y de no trabajo, desaparece un elemental factor de deslinde -físico o geográfico- entre uno y otro, y facetas profesionales y personales y familiares tienden a confundirse y superponerse. Mientras el desplazamiento a las dependencias físicas de la empresa conlleva, una vez en estas el trabajador, el desarrollo exclusivo, por el tiempo pactado, de las funciones laborales encomendadas, al trabajar en el domicilio es frecuente la alternancia de tareas profesionales y personales o familiares 0 domésticas. Salvo estricta autodisciplina del trabajador -lo que exige peculiares condicionantes personales- $u$ otros mecanismos de control remoto -poco frecuentes, al conformar precisamente la flexibilidad un atributo esencial del trabajo a distancia-, la jornada laboral tiende a fragmentarse en periodos cortos entre los cuales se desarrollan tareas no profesionales y el horario, a su vez, acostumbra fluctuar con objeto de atender a cambiantes requerimientos personales y familiares. $Y$ ello afecta sensiblemente a los descansos, desde una perspectiva cuantitativa y, así mismo, cualitativa ${ }^{24}$. Por un lado, estos tienden a mermar: una dedicación entrecortada a las tareas profesionales o en horarios poco convenientes -al final del día o parcialmente en horario nocturno- a menudo implica menor concentración y, por consiguiente, menor rendimiento, lo que, a posteriori, es compensado por un mayor número de horas de trabajo. Por otro lado, tales descansos, más cortos -señaladamente, entre el final de una jornada y el inicio de la segunda-, son, además, cualitativamente peores, al propiciar menor recuperación, física y mental, de los trabajadores que las interrupciones más prolongadas. Y en la calidad de los descansos influyen igualmente las horas del día en que se proyectan, resultando poco propicias horas centrales del día en que familiares u otros convivientes desenvuelven su actividad cotidiana y que, por consiguiente, son más ruidosas.

Además del ya referido registro de jornada, otros preceptos de la norma de 2021 parecen encaminarse a la ordenación del tiempo de trabajo del trabajador a distancia -o, más concretamente, a la distinción de tiempos de trabajo y de descanso-, aún cuando los "mensajes" resultan en ocasiones un tanto contradictorios. Así, en cuanto a horario y tiempo de disponibilidad, uno y otro han de figurar en el acuerdo de trabajo a distancia, al señalar el art. 7 de la Ley 10/2021 que "será contenido mínimo obligatorio del acuerdo de trabajo a distancia, sin perjuicio de la regulación recogida al respecto en los convenios o acuerdos colectivos: c) [el] horario de trabajo de la persona trabajadora y dentro de él, en su caso, [las] reglas e disponibilidad". Lo que sugiere la prefijación de aquellos tramos temporales en que se desarrollará la actividad y, más aún, la identificación de aquellos tiempos, dentro de aquéllos, en que el trabajador se hallará a disposición de la empresa. Pero tal prefijación es, sin embargo, flexible por decisión del trabajador, esto es, mutante o fluctuante, eso sí dentro de los parámetros señalados por el convenio colectivo y el acuerdo de trabajo a distancia y respetando los tiempos de disponibilidad obligatoria y la normativa sobre tiempo de trabajo y descanso (art. 13).

Mención especial merece, finalmente, la regulación del derecho a la desconexión digital de los trabajadores a distancia (art. 18). Pues si bien acostumbra vincularse al derecho a la intimidad o a la conciliación de la vida personal, familiar y laboral, siendo una herramienta de contención del tiempo de trabajo y, correlativamente, de protección de los descansos de los trabajadores, constituye, así mismo, un valioso instrumento de prevención de riesgos laborales ${ }^{25}$. Y este resulta especialmente relevante en el caso de los trabajadores no presenciales cuya mayor exposición a la hiperconexión ya reconoció la Ley Orgánica 3/2018 -primera

\footnotetext{
${ }^{24}$ Deben referirse, en dicho sentido, algunas de las conclusiones plasmadas en el Informe de la OIT (2020), pp. 5-6: “Las investigaciones sobre el teletrabajo han demostrado repetidamente que los empleados que trabajan desde el hogar tienden a trabajar más horas (...); el teletrabajo, en general, puede dar lugar a un horario de trabajo más prolongado y a un aumento de la carga de trabajo durante las noches y los fines de semana (...); los empleados que tienen hijos u otras personas a su cargo en el hogar necesitan encontrar tiempo adicional en su día para realizar su trabajo. Puede que comiencen a trabajar muy temprano en la mañana o continúen hasta mucho más tarde en la noche; también puede que dividan la jornada laboral en segmentos más pequeños, intercalados con descansos para el cuidado de los niños, las tareas domésticas, la educación en el hogar".

${ }^{25}$ No en vano la naturaleza misma del derecho a la desconexión digital, como derecho autónomo, fue en ocasiones cuestionada: MOLINA (2017), pp. 249 y ss.; así como p. 281; VALLECILLO (2017), p. 167.
} 
intervención del legislador español en la materia-, apelando, "en particular", a preservar el derecho a la desconexión digital en los supuestos de realización total o parcial del trabajo a distancia (art. 88.3). Concepción que retoma la norma de 2021 que, con una dudosa técnica legislativa, reitera textualmente el referido art. 88.3 -referencia expresa a los trabajadores a distancia inclusive-, añadiendo con todo un elemento de sumo interés. Incluye una suerte de definición o concreción -la primera- del deber empresarial de garantía del derecho a la desconexión, que, según expresa, ha de conllevar "una limitación del uso de los medios tecnológicos de comunicación empresarial y de trabajo durante los periodos de descanso, así como el respeto a la duración máxima de la jornada y a cualesquiera límites y precauciones en materia de jornada que dispongan la normativa legal o convencional". Siendo obvio tal respeto a la normativa en materia de tiempo de trabajo, no lo es tanto, de ahí su interés, la explícita referencia a una "limitación" del recurso a dispositivos de comunicación "empresarial y de trabajo". Lo cual, si bien no conforma una prohibición, sí apela, por lo demás de modo imperativo, a la contención en la comunicación empresa-trabajador una vez finalizada la jornada de trabajo.

Ciertamente, una intervención más contundente -una prohibición en sentido estrictohabría sido más efectiva. Y no era para menos: los periodos de descanso sobre los que se proyecta la citada "limitación" deben, por su naturaleza misma, resultar inmunes a cualquier género de intromisión por parte del empresario, que, de producirse y determinar una respuesta por parte del trabajador, implica una mutación del periodo de descanso, que pasa entonces a ser de trabajo, con las consiguientes implicaciones en términos de jornada y a efectos retributivos. En cualquier caso, lo señalado conforma al menos un criterio inspirador para las políticas internas y para la negociación colectiva a las que apela el art. 18 de la Ley 10/2021 y que, por tanto, deberían encaminarse, ahora más claramente, hacia la articulación de mecanismos de restricción de las comunicaciones en horario no laboral. Incluso, a falta de tales fórmulas de desarrollo, tal "limitación" conforma ya una suerte de contenido mínimo del derecho a la desconexión digital, ya más tangible y efectivo, al menos para los trabajadores a distancia. En sentido contrario, sin embargo, la Disp. Adic. 1ạ (apartado 2) de la norma de 2021 parece impulsar una negociación flexibilizadora del derecho a la desconexión: conforme aquella, en efecto, convenios o acuerdos colectivos podrán regular "las posibles circunstancias extraordinarias de modulación" de aquél.

\subsubsection{Otros factores: aislamiento, estrategias organizativas y fatiga informática}

No sólo del impacto en la variable tiempo de trabajo derivan riesgos psicosociales. Otra circunstancia prototípica del trabajo a distancia es susceptible de acarrear consecuencias notables en la salud del trabajador: tal es su situación de aislamiento laboral e incluso social. La drástica reducción de los contactos sociales -tal fue, precisamente, el objetivo de la priorización del trabajo a distancia en el marco de la crisis sanitaria- es susceptible de generar trastornos psíquicos ${ }^{26}$. Por ello, superada la pandemia, debiera contemplarse, como opción prioritaria, el trabajo a distancia a tiempo parcial, en alternancia, por tanto, con el desempeño de actividades en las instalaciones de la empresa.

La Ley 10/2021, con todo, sólo impone dicho modelo en el caso de un colectivo especialmente vulnerable -los menores-, así como, en los términos en su caso señalados por la negociación colectiva, de procederse a la contratación en prácticas o para la formación y el aprendizaje (art. 3 y Disp. Adic. 1a). Al margen de este segundo supuesto -en que, con toda probabilidad, los objetivos formativos han determinado la exclusión del trabajo a distancia a jornada completa-, la extensión de la medida a los menores revela su potencial en términos preventivos. Ha de recordarse en dicho sentido que el Anteproyecto configuraba el trabajo a distancia como un trabajo de especial peligrosidad, por tanto, prohibido a las personas menores

${ }^{26}$ Destacando el "riesgo de sentirse excluido" como consecuencia del aislamiento vid., de nuevo, informe de la OIT (2020), p. 13. Sobre el particular, puede así mismo consultarse el Informe de EUROFOUND (2020a), pp. 27 y ss. 
de edad. Prohibición que finalmente no recoge el texto definitivo que opta por su sustitución por la exigencia de una presencialidad mínima del 50 por 100, de este modo configurada como fórmula para la protección de la seguridad y salud de los menores de 18 años-como es lógico, habida cuenta dicha naturaleza, no disponible por la negociación colectiva-.

Ya en cuanto al resto de trabajadores, si bien la norma contempla el trabajo a distancia a jornada completa, acuerdo de trabajo y convenio colectivo pueden establecer la alternancia con la presencialidad. Esta última, en efecto, se configura como eventual contenido mínimo del primero -art. 7.d)- y materia negociable a nivel colectivo -apartado 2 de la Disp. Adic. 1á-, a cuyos efectos debiera tenerse en cuenta el impacto en la salud de los trabajadores de modelos de trabajo exclusivamente a distancia. En cualquier caso, convendría, así mismo, como de hecho ya prevé el Acuerdo Marco de 2002, establecer cauces para el mantenimiento de un contacto regular del trabajador a distancia con el empleador, con otros compañeros de trabajo, así como con la empresa misma ${ }^{27}$. Contacto, de carácter personal, que coadyuvaría a paliar los riesgos derivados del aislamiento y que, sin embargo, no contempla la Ley 10/2021.

Y los riesgos a menudo surgen de la opción por determinadas estrategias organizativas: así, de recurrir el empresario a la organización del trabajo "según un cierto ritmo". Método que ya genera la advertencia del legislador español, que apela a la consideración del "principio general de adaptación del trabajo a la persona, especialmente de cara a atenuar el trabajo monótono y repetitivo" -art. 36.5 ET-, sin duda para procurar contrarrestar faltas de concentración y evitar despistes a menudo desencadenantes de daños. Pero debe, igualmente, ponderarse el impacto psicosocial del trabajo según un cierto ritmo: pues, tanto la monotonía o aburrimiento como la exigencia de realización de un determinado número de tareas en el lapso de tiempo prefijado -escaso, habida cuenta el volumen de aquéllas- pueden derivar en daños psicosociales. En dicho sentido, a los riesgos organizacionales de quienes trabajan con ordenadores, parece referirse, aún de modo muy incipiente, el Real Decreto 488/1997, al condicionar lo que denomina "Interconexión ordenador/persona" a los siguientes requisitos básicos (apartado 3 del Anexo): que el programa empleado se adapte a la tarea que deba realizarse, que sea fácil de usar conforme al nivel de conocimientos y de experiencia del usuario, que los sistemas proporcionen a los trabajadores indicaciones sobre su desarrollo y muestren la información en un formato y a un ritmo adaptados a los operadores, o, en términos más generales, que "los principios de ergonomía resulten aplicables al tratamiento de la información por parte de la persona". Ello, sin duda, también debiera ser objeto de una reforma modernizadora, conformando la referencia explícita a los "riesgos organizativos" -art. 16.1 de la Ley 10/2021- un óptimo punto de partida.

Y ya se ha referido la "carga mental" -según la terminología del Real Decreto 488/1997generada, especialmente en el caso de los teletrabajadores, por el manejo muy intenso de equipos o instrumentos informáticos. "Carga mental" o, conforme a las normas más recientes, "fatiga informática", que cabría definir como el cansancio, hastío o agotamiento, fundamentalmente de carácter psicológico -sin perjuicio, por supuesto, de su eventual somatización-, derivado del recurso a herramientas informáticas, sobre las que, por otro lado, el trabajador acostumbra desarrollar una valoración negativa. Sobre este riesgo ya advirtió la Ley Orgánica 3/2018, a cuyo tenor "el empleador (...) elaborará una política interna dirigida a trabajadores, incluidos los que ocupen puestos directivos, en la que definirán [además de las modalidades de ejercicio del derecho a la desconexión] las acciones de formación y de sensibilización del personal sobre un uso razonable de las herramientas tecnológicas que evite el riesgo de fatiga informática" (art. 88.3), prosiguiendo la norma con una referencia expresa a quienes prestan servicios, total o parcialmente, a distancia, que, por consiguiente, el legislador entiende más expuestos a la hiperconexión -de ahí la referencia explícita, ya comentada, a su derecho a desconectar-, pero también, como es lógico, a la citada "fatiga informática". Y todo ello, como ya se apuntó, de nuevo es objeto de reiteración por la Ley 10/2021 (art. 18.2, en términos idénticos al referido art. 88.3).

${ }^{27}$ Apartado 9 del Acuerdo Marco Europeo. 
La "fatiga informática", con todo, presenta muy probablemente un matiz diferente a la "carga mental" referida por la norma de finales de los 90, atendiendo al peculiar impacto de las TICs en la salud del trabajador. A su manejo en particular se vinculan, en efecto, los siguientes trastornos específicos: la "tecnofatiga", la "tecnoansiedad" y la "tecnoadicción", manifestaciones del fenómeno, más amplio, del "tecnoestrés" ${ }^{28}$. Concurren en la tecnofatiga "sentimientos de cansancio y agotamiento mental y cognitivo" debidos al uso de TICs, respecto de las cuales, además, el sujeto desarrolla actitudes escépticas y creencias de ineficacia. Ya en el caso de la tecnoansiedad, el trabajador experimenta "tensión y malestar por el uso presente o futuro de algún tipo de TIC", lo que, de nuevo, le conduce a generar "pensamientos negativos", en este caso, sobre su capacidad y competencia en el manejo de las TICs. Incluso, en supuestos más extremos, se ha detectado un tipo específico de tecnoansiedad, la "tecnofobia", en que el sujeto experimenta miedo ante las TICs y desarrolla "sentimientos hostiles o agresivos" hacia estas. De signo contrario, finalmente, es la tecnoadicción: de nuevo una subcategoría de estrés tecnológico, pero en la que el trabajador no manifiesta rechazo, aprensión o recelo hacia las TICs, sino una incontrolable compulsión a su utilización en cualquier tiempo y lugar y por periodos muy prolongados, desarrollando una verdadera dependencia de aquéllas ${ }^{29}$.

Sin duda, la contención de los tiempos de manejo de equipos informáticos y de las TICs, así como la correlativa salvaguarda de los descansos se erigen en herramientas óptimas para evitar carga mental y fatiga informática. Por lo que merecen una valoración positiva medidas como la fijación del horario en el acuerdo de trabajo a distancia, el registro de jornada o la concreción del derecho a la desconexión. Con todo, deben recordarse aquí las lagunas más arriba señaladas.

\subsection{Una necesaria perspectiva de género}

El trabajo a distancia presenta una evidente carga de género ${ }^{30}$. A menudo, en efecto, el recurso al trabajo no presencial deriva de una necesidad muy acuciante de atención de obligaciones familiares. Recurso, por lo demás, en España, expresamente fomentado por el legislador ex art. 34.8 ET -tras la reforma operada por Real Decreto-ley 6/2019- en línea con la Directiva 2019/1158 (arts. 3.f) y 9), pero también, en el caso específico del teletrabajo, con el Acuerdo Marco de 2002. Lo que, dada la mayor predisposición de las trabajadoras a adaptar su modo de trabajo a las necesidades de su familia -por razones culturales, sociales y también económicas- permite augurar una feminización del trabajo a distancia con vocación conciliadora. Pero al margen incluso de tal opción deliberada, la modalidad de trabajo no presencial, aún forzada o padecida -señaladamente, habida cuenta la priorización operada en el marco de la pandemia- implica a menudo una alteración del reparto de tareas domésticas y de cuidado entre quien trabaja en casa -que tiende a una mayor asunción de las mismas- y quien lo hace en las instalaciones de la empresa -en cambio, más liberado-. Y más mujeres que hombres han iniciado un desarrollo a distancia de su prestación laboral como consecuencia de la pandemia ${ }^{31}$. En ambos casos, por tanto, y con la referida feminización, es probable un impacto muy notable en términos profesionales -al incidir la combinación de tareas laborales y familiares en la formación y promoción de quien trabaja a distancia-, pero también desde una perspectiva de prevención de riesgos laborales.

$Y$ es que el ya referido deslinde entre tiempo de trabajo y tiempo de descanso se torna más complejo aún en el caso del trabajo a distancia con vocación de conciliación. Supuesto en que el trabajo a distancia, concebido como "fórmula de trabajo flexible" -en la terminología de la Directiva europea-, constituye una opción deliberada del trabajador con objeto precisamente

\footnotetext{
${ }^{28}$ Clasificación extraída de la Nota Técnica 730 "Tecnoestrés: concepto, medida e intervención psicosocial” del Instituto Nacional de Seguridad e Higiene en el Trabajo. En términos similares, MELLA (2017), pp. 73-74.

${ }^{29}$ Nota Técnica 730 .

${ }^{30}$ Sobre el particular, FERNÁNDEZ (2019), pp. 575 y ss.

${ }^{31}$ Informe de EUROFOUND (2020b), p. 21.
} 
de armonizar trabajo y familia. Pues, el trabajo a distancia, ciertamente, puede contribuir a la conciliación: permite, por un lado, optimizar el tiempo, ahorrando aquel invertido en el desplazamiento del hogar al trabajo -y viceversa-, y conlleva, así mismo, un mayor margen de selección de aquellos tramos temporales dedicados a la actividad profesional -en estas circunstancias, precisamente aquellos en que no deben atenderse obligaciones familiares-. Pero ello también genera riesgos para la salud del trabajador a distancia: en primer término, como ya se ha apuntado, por la excesiva fragmentación de tiempos que acostumbra producirse y por no ser estos igualmente idóneos para trabajar y descansar, pero también por la frecuente superposición de tiempos de trabajo y de no trabajo, conjugando el trabajador -muy a menudo la trabajadora- tareas laborales y no laborales, por ejemplo, de cuidado. Y el esquema se ha reproducido en el marco del trabajo a distancia generado por la pandemia -en realidad, forzado por las circunstancias-, con el agravante añadido del cierre de los centros educativos y de atención a dependientes y el correlativo incremento sustancial de las tareas de cuidado de niños y mayores.

A ello atiende la nueva norma, que descarta la idea de que el tiempo de trabajo y el tiempo de cuidado pueden ser simultáneos. Lo que se traduce -art. 4.5- en el explícito reconocimiento a los trabajadores a distancia de los "mismos derechos que las personas trabajadoras presenciales en materia de conciliación y corresponsabilidad, incluyendo el derecho de adaptación a la jornada establecido en el art. 34.8 ET, a fin de que no interfiera el trabajo con la vida personal y familiar". De nuevo, la advertencia se estima positiva: pone el foco de atención en muchas trabajadoras que ya no desempeñan sucesivamente la "clásica" doble jornada, sino que, tras retornar al hogar, se han convertido en trabajadoras-cuidadoras que desarrollan a un tiempo, funciones profesionales y de crianza o de atención a personas dependientes.

En términos estrictamente jurídicos, con todo, la respuesta merece alguna reflexión: el reconocimiento legal de los derechos en materia de conciliación y corresponsabilidad poco añade, pues, de hecho, ninguna norma excluía a los trabajadores a distancia de su ámbito de aplicación. Y debe lamentarse el mantenimiento de la dicción literal del art. 34.8 ET, a cuyo tenor, como se recordará, las personas trabajadoras tienen derecho a solicitar la prestación de su trabajo a distancia, precisamente "para hacer efectivo su derecho a la conciliación de la vida familiar y laboral". De modo que el trabajo a distancia conforma aún, pese a su impacto de género, una herramienta legal de conciliación, omitiendo el referido art. 34.8 ET toda precisión relativa a un uso adecuado de la misma. Sin duda, tratándose de un potencial factor de discriminación por razón de sexo una configuración más matizada habría sido deseable. Al igual que otras modalidades de contratación en la práctica erigidas en instrumento de conciliación tal es el caso del trabajo a tiempo parcial-, el trabajo a distancia, padecido y condicionado por la necesidad de conjugar trabajo y familia, constituye un nuevo foco de segregación, por lo que aquí interesa también con repercusiones notables en la salud de las trabajadoras. La atención de obligaciones familiares potencia especialmente aquellos riesgos inherentes a la ordenación del tiempo de trabajo del trabajador a distancia: la jornada, más entrecortada aún, se alarga, lo que, a su vez, implica menores y peores descansos, y la superposición de tareas genera ansiedad $y$, a menudo, insatisfacción en ambas facetas vitales -al estimarse muchas veces, una y otra, indebidamente atendidas-.

En dicho sentido, la regulación del tránsito, de la presencialidad al trabajo a distancia, ofrecida por la norma de 2021 resulta más adecuada. Habilitados convenios o acuerdos colectivos para regular los mecanismos y criterios de transición al trabajo a distancia, así como para articular preferencias atendiendo a las circunstancias aducidas -entre otras, personales o familiares-, la Ley 10/2021 incluye una advertencia que, sin duda, debería así mismo albergar el ET: “En el diseño de estos mecanismos se deberá evitar la perpetuación de roles y estereotipos de género y se deberá tener en cuenta el fomento de la corresponsabilidad entre mujeres y hombres, debiendo ser objeto de diagnóstico y tratamiento por parte del plan de igualdad que, en su caso, corresponda aplicar en la empresa" (art. 8.3). Con todo, la materialización práctica 
de dicho mandato a los negociadores, desde la óptica de los principios de igualdad y no discriminación por razón de sexo, sin duda, deberá examinarse con sumo rigor.

\section{La barrera "física": la proyección de la normativa preventiva en el domicilio u otro lugar designado por el trabajador}

La evaluación de riesgos, la aplicación de las medidas de seguridad y la verificación de su efectivo cumplimiento requieren, a priori, de un acceso directo al lugar de trabajo. Por lo que, hallándose este en el domicilio del trabajador a distancia u otro emplazamiento fuera del centro de trabajo, surgen evidentes dificultades prácticas ${ }^{32}$. Por ello, ya el Acuerdo Marco de 2002 estableció, "dentro de los límites de la legislación y los convenios colectivos nacionales", el derecho de acceso del empresario, de los representantes de los trabajadores y/o de las autoridades competentes al lugar de trabajo, de ser este el domicilio del trabajador, "previa notificación y consentimiento previo" (Cláusula Octava). Una cuestión a la que es sensible la norma de 2021, que, además, de erigir el lugar de prestación de servicios en contenido mínimo del acuerdo de trabajo a distancia (art. 7.f) ${ }^{33}$, dedica a esta temática una parte sustancial de la Sección -la 4a del Capítulo III- específicamente dedicada a la prevención de riesgos laborales.

El acceso al lugar de trabajo por razones de prevención, sin embargo, ni se configura como necesario en todos los escenarios de trabajo a distancia -no es siquiera la opción prioritaria-, ni es, de estimarse la necesidad de actuación in situ, automático. En efecto, conforme dispone el art. 16.2 de la Ley 10/2021, a modo de regla general, la empresa ha de obtener toda la información relativa a riesgos a los que esté expuesto el trabajador a distancia "mediante una metodología que ofrezca confianza respecto de sus resultados", adoptando a continuación las medidas de protección correspondientes. Y ya con carácter subsidiario -cuando la obtención de dicha información exija la visita al lugar de trabajo por parte de quien tenga competencias en materia preventiva-, procederá la emisión de informe escrito que justifique dicha circunstancia y que se entregará a los delegados de prevención y al trabajador a distancia. Pero ello tampoco determinará siempre un acceso al lugar de trabajo: pues, cuando este se desempeñe en el domicilio de la persona trabajadora o en el de otra persona física, la visita requerirá, en cualquier caso, permiso del trabajador. $Y$, de no concederse este, la actividad preventiva habrá de desarrollarse con base en la identificación de los riesgos derivada de la información proporcionada por el trabajador conforme a las instrucciones del servicio de prevención. Además, el referido acceso, en los términos indicados, queda expresamente restringido a "la zona habilitada para la prestación de servicios, no extendiéndose al resto de zonas de la vivienda o del lugar elegido para el desarrollo del trabajo a distancia".

De este modo, el Derecho interno aborda al fin el acceso por razones de prevención al lugar de trabajo del trabajador a distancia. Sin embargo, en el necesario proceso de ponderación de los derechos en conflicto -por un lado, el derecho a la seguridad y salud laboral y, por otro, el derecho a la intimidad personal y familiar- el esquema legal descrito parece priorizar este último. Si bien la circunscripción del acceso a la exclusiva zona habilitada para trabajar y la exigencia de permiso a efectos de acceso a viviendas particulares se hallan del todo fundamentados, la configuración de la visita como opción subsidiaria, y no general, o la omisión de una posible solicitud de acceso por parte del trabajador son más criticables. Ello muy probablemente determine, en la mayoría de los casos, la asunción por el trabajador a distancia de las obligaciones de evaluación de riesgos y de aplicación de las medidas de seguridad. Una opción que, a nuestro entender, ofrece escasas garantías del efectivo cumplimiento de la normativa preventiva. Pues aun cuando se proporcione al trabajador una sólida formación previa, así como una detallada documentación de apoyo -en que se especifiquen los elementos objeto de comprobación y que debe ser objeto de cumplimentación rigurosa-, al pender el proceso exclusivamente del propio trabajador, resulta imposible seguimiento alguno. A falta de

${ }^{32}$ Destacando esto último CRUZ (2020b), p. 409, así como ÁlVAREZ (2020), p. 189.

${ }^{33}$ Lo que subsana una laguna del anterior art. $13 \mathrm{ET}$. 
verificación externa, por tanto, la prevención se reconduce, en esencia, a las referidas formación y declaración del interesado, revelando en su caso posteriores exámenes médicos, ex art. 22 LPRL, eventuales daños en su salud. Así, aún tras la reforma, mientras en el trabajo presencial las obligaciones de los trabajadores en materia preventiva -señaladas por los arts. 5.b) ET y 29 LPRL- se reconducen en esencia al estricto cumplimiento de las medidas arbitradas por el empresario, la elección del lugar de trabajo determina la asunción por el trabajador a distancia de un rol más activo. Toda vez que de su colaboración pende, en la mayoría de las ocasiones, la puesta en práctica y efectividad de las obligaciones empresariales en materia preventiva, el trabajador a distancia ha de asumir un papel proactivo. Pues, se halla implicado en el proceso mismo de diagnóstico de situación -la evaluación de los riesgos laborales-, así como en el de implementación de las medidas de seguridad, a menudo con muy escasa, o incluso nula, intervención de terceros.

\section{Conclusiones}

La investigación desarrollada permite extraer las conclusiones que siguen.

Es muy probable, en primer término, que el trabajo no presencial ocupe en el futuro un lugar más destacado que en tiempos pasados. Tras el macro-ensayo operado en los últimos meses, puede augurarse, inclusive una vez superada la pandemia, no sólo un mayor recurso al trabajo a distancia, sino también la implementación, más generalizada, de fórmulas mixtas. Por ello resultaba perentoria una intervención legislativa en la materia, una reforma garantista de los derechos de los trabajadores, que atajase el proceso de conversión del trabajo a distancia en potente foco de precarización de condiciones de trabajo, también de las de seguridad y salud.

Conviene tener presente, en segundo lugar, el carácter ambivalente del trabajo a distancia: pues, si bien presenta notables ventajas -en clave empresarial, a los ojos de los trabajadores y en términos colectivos-, los riesgos vinculados a tal modo de prestación de servicios no son en absoluto menores. En particular, la flexibilidad horaria, inherente al trabajo a distancia y cualidad estrella perseguida por empleadores y empleados, merece un tratamiento especialmente cauteloso. La misma, en efecto, a menudo deriva en prácticas especialmente nefastas para la salud de los trabajadores: sin horario laboral concreto, con el único compromiso de realización o entrega de las tareas pactadas, tiempo de trabajo y de descanso confluyen y se confunden. Incluso, de concurrir necesidades de conciliación, a menudo motor del tránsito al trabajo a distancia, tareas laborales y de cuidado se superponen, desarrollando a un tiempo las trabajadoras la tradicional doble jornada, ahora convertida en una suerte de "jornada simultánea". Y debe repararse así mismo en el aislamiento, laboral y a menudo social, característico del trabajo a distancia. Por si fuera poco, el teletrabajo -hoy por hoy, manifestación prioritaria del trabajo a distancia- suma riesgos particulares: aquellos derivados del manejo intenso -a menudo, a lo largo de toda la jornada- de medios informáticos, pero también, de manera específica, de las TICs -vehículo de transmisión del poder de dirección de la empresa ahora con capacidad de proyección en cualquier lugar y a cualquier hora-.

A modo de tercera conclusión, conviene destacar que la reciente Ley española de trabajo a distancia incorpora previsiones que merecen favorable acogida. Debe subrayarse el esfuerzo de visibilización de los factores "psicosociales, ergonómicos y organizativos" concurrentes en el trabajo a distancia, la implementación de herramientas de deslinde entre tiempo de trabajo y de descanso -como es el caso del registro horario- y por supuesto la muy necesaria advertencia de que las fórmulas de tránsito al trabajo a distancia deben "evitar la perpetuación de roles y estereotipos de género". En el tintero, sin embargo, han quedado actuaciones no menores: entre otras, una configuración más concreta del derecho a la desconexión digital, una apuesta más decidida por fórmulas mixtas de semi-presencialidad y, ya en vía reglamentaria, la fijación de aquellas condiciones mínimas de seguridad y salud exigibles, aún en el domicilio o lugar designado por el trabajador, a entornos laborales y equipos de trabajo. 
Debe apelarse, finalmente, a una intervención decidida de la negociación colectiva. En el plano colectivo y desde una perspectiva preventiva, sería muy deseable la identificación de aquellas actividades o puestos susceptibles de desempeño a distancia -o, por el contrario, vetados a ello-, el establecimiento de jornadas presenciales mínimas u otras fórmulas de contacto directo entre trabajadores a distancia y empresa, la materialización práctica del derecho a la desconexión, así como, por supuesto, la articulación de herramientas de fomento de un recurso corresponsable al trabajo a distancia -contrarrestando la actual sobreexposición de las trabajadoras a los riesgos inherentes a este último-.

\section{BIBLOGRAFÍA CITADA}

Álvarez Cuesta, HenAR (2020): “Del recurso al teletrabajo como medida de emergencia al futuro del trabajo a distancia”, en: Lan Harremanak (№ 43), pp. 175-201.

CASAS BAAMONDE, MARÍA EMILIA (2020): “El carácter preferente del trabajo a distancia en el estado de alarma y en la crisis sanitaria", en: Derecho de las Relaciones Laborales (№ 7), pp. 921-936.

Casas BaAmonde, María Emilia y Rodríguez-Piñero Bravo-Ferrer, Miguel (2020): “Un nuevo Derecho del Trabajo en la emergencia. Las medidas laborales y de seguridad social excepcionales en el estado de alarma declarado por la crisis sanitaria de Covid-19", en: Derecho de las Relaciones Laborales (№ 4), pp. 317-348.

COMISIÓN DE EXPERTOS EN APLICACIÓN DE CONVENIOS Y ReCOMENDACIONES (2020): “Informe: Promover el empleo y el trabajo decente en un panorama cambiante". Disponible en https://www.ilo.org/ilc/ILCSessions/109/reports/reports-to-the-

conference/WCMS_738283/lang--es/index.htm [visitado el 10 de mayo de 2021].

CRUz Villalón, Jesús (2020a): "Del coronavirus al contagio del teletrabajo". Disponible en: http://jesuscruzvillalon.blogspot.com/2020/03/del-coronavirus-al-contagio-del.html [visitado el 10 de mayo de 2021].

CRUz VILlalón, Jesús (2020b): "Teletrabajo y coronavirus: de la emergencia a la permanencia”, en: Derecho de las Relaciones Laborales (№ 4), pp. 406-419.

De Las Heras García, Aránzazu (2016): El teletrabajo en España: un análisis crítico de normas y prácticas (Madrid, CEF).

EUROFOUND (2020a): "Informe: Telework and ICT-based mobile work: Flexible working in the digital age (Luxemburgo, Eurofound)". Disponible en: https://www.eurofound.europa.eu/sites/default/files/ef_publication/field_ef_document/ef1903 2en.pdf [visitado el 10 de mayo de 2021].

EUROFOUND (2020b): "Informe: Living, working and COVID-19 (Luxemburgo, Eurofound)". Disponible en: https://www.eurofound.europa.eu/sites/default/files/ef_publication/field_ef_document/ef2005 9en.pdf [visitado el 10 de mayo de 2021].

Fernández Prol, Francisca (2019): “Teletrabajo en clave de género: ¿herramienta de corresponsabilidad o foco de segregación?", en: Asociación Española de Derecho del Trabajo y de la Seguridad Social, XXIX Congreso Anual de la AEDTSS - El futuro del trabajo. Cien años de la OIT (Madrid, Ministerio de Empleo y Seguridad Social), pp. 575-594.

FernÁndez ProL, Francisca (2020): “Tiempos de vida y de trabajo: el impacto de las TICs", en: Temas Laborales (№ 151), pp. 259-274.

IGUARTUA MIRÓ, MARÍA TERESA (2019): “La obligación de registro de la jornada de trabajo tras el RDL 8/2019”, en: Temas Laborales (№ 147), pp. 115-149. 
Mella Méndez, lourdes (Ed.) y Villalva Sánchez, Alicia (CoORd.) (2015): Trabajo a distancia y teletrabajo. Estudios sobre su régimen jurídico en el Derecho español y comparado (Navarra, Aranzadi).

MELLA MÉNDEZ, LOURDES (DIR.) (2017): El teletrabajo en España: aspectos teórico-prácticos de interés (Madrid, Wolters Kluwers).

Molina NAVARRETE, CRIStÓBAL (2017): "Jornada laboral y tecnologías de la info-comunicación: la 'desconexión digital', garantía del derecho al descanso", en: Temas Laborales (№ 138), pp. 249283.

OIT (2020): "Informe: El teletrabajo durante la pandemia de COVID-19 y después de ella (Ginebra, OIT)". Disponible en: https://www.ilo.org/wcmsp5/groups/public/---ed_protect/---protrav/--travail/documents/publication/wcms_758007.pdf [visitado el 10 de mayo de 2021].

SAlA Franco, Tomás (COORD.) (2020): El teletrabajo (Valencia, Tirant Lo Blanch).

SieRra Benítez, EsPeranza MACARENa (2011): El contenido de la relación laboral en el teletrabajo (Sevilla, CES Andalucía).

Thibault ARANDA, JAVIer (2001): El teletrabajo. Análisis jurídico-laboral, 2a edición (Madrid, CES).

USHAKOVA, TANIA (2019): “Convenio sobre el trabajo a domicilio 1996 (núm. 177)”, en: Revista Internacional y Comparada de Relaciones Laborales y Derecho del Empleo (Vol. 7, Núm. Extra 0), pp. 1364-1379.

Vallecillo Gámez, María Rosa (2017): “El derecho a la desconexión: ¿« «novedad digital» o esnobismo del «viejo» derecho al descanso?", en: Revista de Trabajo y Seguridad Social, CEF (№ 408), pp. 167-178.

\section{JURISPRUDENCIA CITADA}

STJCE de 1 de diciembre de 2005, Asunto C-14/04, Abdelkader Dellas.

STJUE de 10 de septiembre de 2015, Asunto C-266/14, Tyco.

\section{NORMAS JURÍDICAS CITADAS}

DIRECTIVA 1989/391/CEE, de 12 de junio, para la aplicación de medidas para promover la mejora de la seguridad y de la salud de los trabajadores en el trabajo. DOCE, 29 de junio de 1989.

DIRECTIVA 2003/88/CE, del Parlamento Europeo y del Consejo, de 4 de noviembre de 2003, relativa a determinados aspectos de la ordenación del tiempo de trabajo. DOUE, 18 de noviembre de 2003.

DIRECTIVA (UE) 2019/1158, de 20 de junio de 2019, relativa a la conciliación de la vida familiar y la vida profesional de los progenitores y los cuidadores. DOUE, 12 de julio de 2019.

CONSTITUCIÓN ESPAÑOLA de 6 de diciembre de 1978. BOE, 21 de diciembre de 1978.

LEY 31/1995, de 8 de noviembre, de prevención de Riesgos Laborales. BOE, 10 de noviembre de 1995.

LEY 10/2021, de 9 de julio, de trabajo a distancia. BOE, 10 de julio de 2021.

ReAl DeCRETo 486/1997, de 14 de abril, por el que se establecen las disposiciones mínimas de seguridad y salud en los lugares de trabajo. BOE, 23 de abril de 1997.

REAL DECRETO 488/1997, de 14 de abril, sobre disposiciones mínimas de seguridad y salud relativas al trabajo con equipos que incluyen pantallas de visualización. BOE, 23 de abril de 1997. 
REAL DeCRETO 773/1997, de 30 de mayo, en materia de equipos de protección individual. BOE, 12 de junio de 1997.

REAL DeCRETO 1215/1997, de 18 de julio, relativo a la utilización de los equipos de trabajo. BOE, 7 de agosto de 1997.

REAL DECRETO 2/2015, de 23 de octubre, por el que se aprueba el texto refundido de la Ley del Estatuto de los Trabajadores. BOE, 24 de octubre de 2015.

Ley ORgánica 3/2018, de 5 de diciembre, de Protección de Datos Personales y garantía de los derechos digitales. BOE, 6 de diciembre de 2018.

REAL DECRETO-LEY 6/2019, de 1 de marzo, de medidas urgentes para garantía de la igualdad de trato y de oportunidades entre mujeres y hombres en el empleo y la ocupación. BOE, 7 de marzo de 2019.

REAL DECRETO-LEY 8/2019, de 8 de marzo, de medidas urgentes de protección social y de lucha contra la precariedad laboral en la jornada de trabajo. BOE, 12 de marzo de 2019.

REAL DECRETO-LEY 8/2020, de 17 de marzo, de medidas urgentes extraordinarias para hacer frente al impacto económico y social del COVID-19. BOE, 18 de marzo de 2020.

ReAl DeCRETo-Ley 28/2020, de 22 de septiembre, de trabajo a distancia. BOE, 23 de septiembre de 2020. 